\title{
Electrical Stimulation Frequency and Skeletal Muscle Characteristics: Effects on Force and Fatigue
}

\author{
Maria Vromans (1), Pouran Faghri $(1,2)$ \\ (1) Department of Biomedical Engineering, University of Connecticut, Storrs, CT, USA (2) \\ Department of Allied Health Sciences, Storrs, CT, USA. \\ This article is distributed under the terms of the Creative Commons Attribution Noncommercial License (CC BY-NC 4.0) which permits \\ any noncommercial use, distribution, and reproduction in any medium, provided the original author(s) and source are credited.
}

\begin{abstract}
This investigation aimed to determine the force and muscle surface electromyography (EMG) responses to different frequencies of electrical stimulation (ES) in two groups of muscles with different size and fiber composition (fast- and slow-twitch fiber proportions) during a fatigueinducing protocol. Progression towards fatigue was evaluated in the abductor pollicis brevis (APB) and vastus lateralis (VL) when activated by ES at three frequencies (10, 35, and $50 \mathrm{~Hz})$. Ten healthy adults (mean age: $23.2 \pm 3.0$ years) were recruited; participants signed an IRB approved consent form prior to participation. Protocols were developed to 1) identify initial ES current intensity required to generate the $25 \%$ maximal voluntary contraction (MVC) at each ES frequency and 2) evaluate changes in force and EMG activity during ES-induced contraction at each frequency while progressing towards fatigue. For both muscles, stimulation at $10 \mathrm{~Hz}$ required higher current intensity of ES to generate the initial force. There was a significant decline in force in response to ES-induced fatigue for all frequencies and for both muscles $(p<0.05)$. However, the EMG response was not consistent between muscles. During the progression towards fatigue, the APB displayed an initial drop in force followed by an increase in EMG activity and the VL displayed a decrease in EMG activity for all frequencies. Overall, it appeared that there were some significant interactions between muscle size and fiber composition during progression towards fatigue for different ES frequencies. It could be postulated that muscle characteristics (size and fiber composition) should be considered when evaluating progression towards fatigue as EMG and force responses are not consistent between muscles.
\end{abstract}

Key Words: electrical stimulation, muscle fatigue, electromyography

Eur J Transl Myol 27 (3): 239-245

Fatigue is defined as drop in force from pre-to -post repeated ES-induced muscle contraction protocols ${ }^{1,2}$ or declines in ES-induced torque., ${ }^{3,4}$ However, EMG changes during fatiguing ES protocols are not consistent and have shown both increases or decreases in electrical activity, defined by root-mean-square (RMS) amplitude, peak-to-peak amplitude and area under the signal. ${ }^{3,5}$ Yet, with frequency assessment, research more consistently observed decreases in mean frequency (MNF) and median frequency (MDF) as fatigue progressed. ${ }^{6,7}$ Furthermore, effectively monitoring EMG activity of the muscle throughout the duration of ES also creates added complexity. This is due to ESinduced contractions cluttering the pure EMG signals, masking the unique muscle activity of the signals. These have been attributed to either close proximity of ES electrodes to the EMG detection region or other external noises. ${ }^{5,8}$ Evidently, these challenges limited researchers' abilities to establish a clear understanding of muscle responses to ES and fiber recruitment pattern with ES. Many approaches have been proposed for artifact removal including software filters incorporating wavelet transformations, ${ }^{9}$ hardware devices that employ switch circuits to shut down amplifier control, ${ }^{10}$ and more recently a new approach called Empirical Mode Decomposition (EMD). EMD implements a repeated sifting process that identifies local maxima and minima in the signal to form envelopes that are extracted as 

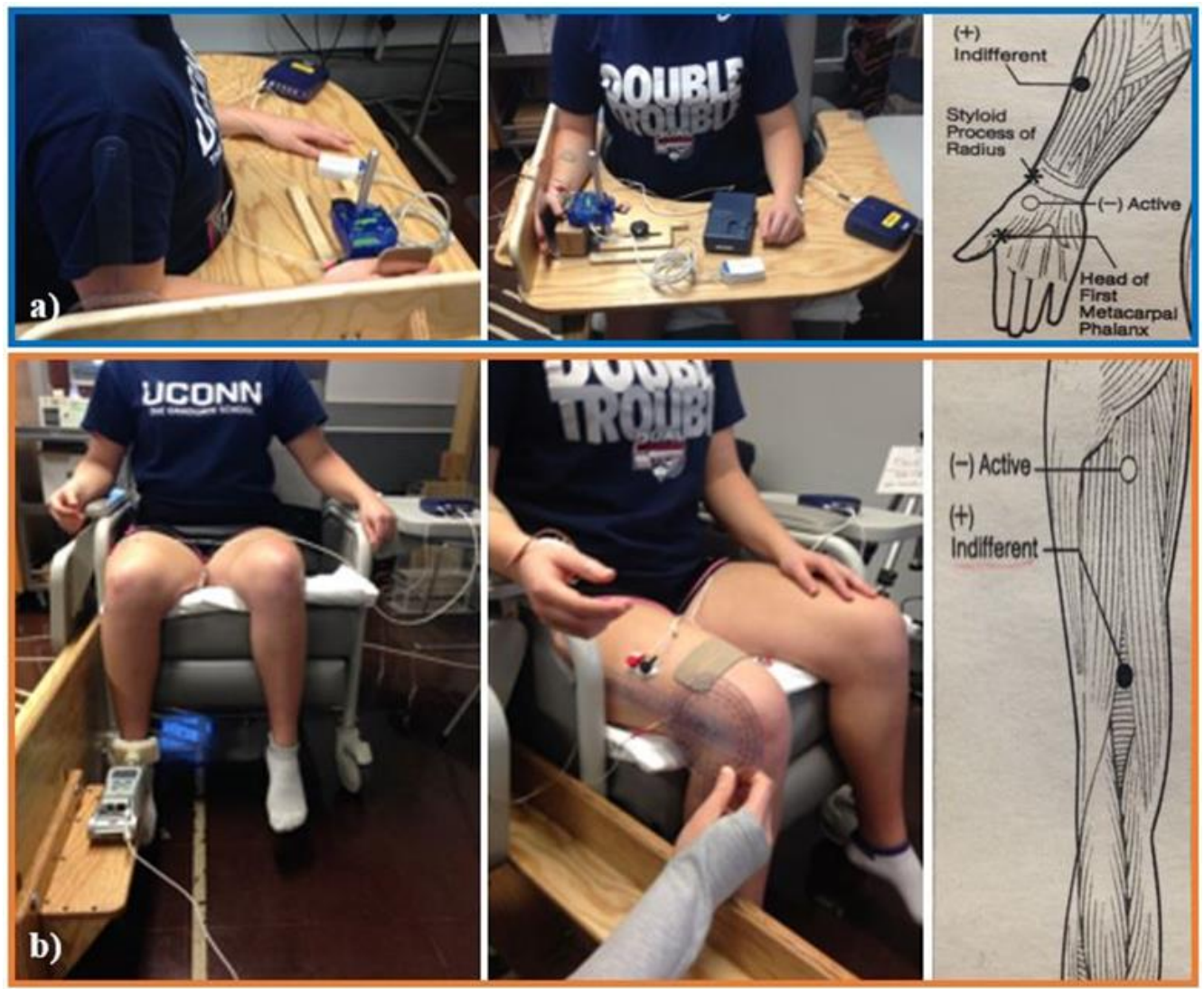

Fig 1. Experimental setup for the (a) APB and (b) VL.

intrinsic mode functions (IMFs) in order to separate muscular components from the artifacts. ${ }^{9,11}$

This application seems to be more promising and was applied to filtering EMG signals in the present study. Moreover, muscles of different size and fiber composition may respond differently to distinctive ES frequency and current intensity and such characteristics may also play a role in the development of fatigue. Understanding these characteristics may help in the development of more effective ES protocols that delay fatigue while maximizing muscle response during rehabilitation treatment. For example, research has suggested that larger muscles, like the predominantly type II vastus lateralis, ${ }^{12}$ generally favor the recruitment of additional motor units as a means of increasing force production until the force demand ranges from $60-90 \%$ MVC; thereafter, these muscles rely on greater firing rates to achieve additional force. On the contrary, smaller muscles, such as the predominantly type I abductor pollicis brevis (APB), ${ }^{12}$ utilize the recruitment of additional motor units until force production nears $\sim 30 \% \mathrm{MVC}$, after which increases in firing rate enhance force production. ${ }^{13}$ Related response variability may be true during ES stimulation when selecting the frequency of ES firing for the purpose of either force generation or improving muscle integrity and rehabilitation training. Several studies have examined how manipulation of stimulation frequency, current intensity, and pulsewidth parameters impact ES-induced fatigue, ${ }^{14-18}$ but to our knowledge little research has examined the development of fatigue with consideration of combined effects of muscle sizes with fiber composition variability in the same group of subjects. Additionally, more recent interest has directed ES application towards tissue regeneration where research has shown that ES aids cell proliferation in connective tissue, improves the rate of new collagen formation in injured tendons, ${ }^{19}$ and reverses long-term denervation muscle atrophy. ${ }^{20}$ Therefore, it is important to understand how ES affects different types of fibers in order to tailor the stimulation to specific muscle types to enhance tissue growth as well as healing while promoting functional movements. The purpose of this investigation was to evaluate the effect of different ES frequencies $(10,35$, and $50 \mathrm{~Hz})$ on the development of muscle fatigue in muscles of different size and fiber composition (APB and VL) utilizing ES current intensity, force, and EMG activity as outcome measures. We hypothesized that fatigue response to ES-induced contractions will be different between muscles and will depend on ES characteristics. Accordingly, muscle size and fiber composition should be carefully planned when developing ES-induced muscle contraction protocols. 


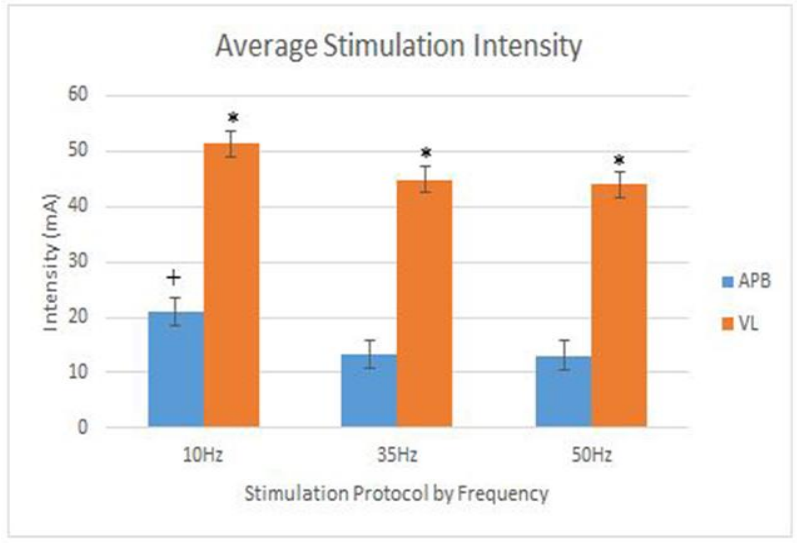

Fig 2. Required stimulation intensity to achieve initial contractile force (25\%MVC). (*) denotes significant differences between muscles $(\mathrm{p}<0.05)$. (+) denotes significant differences within muscle $(\mathrm{p}<0.05)$.

\section{Materials, Methods}

\section{Participants}

Ten healthy male and female participants $(23.2 \pm 3.0$ years) with no known history of musculoskeletal or cardiovascular problems, or known allergy to surface electrodes or adhesive tape were recruited. Full-board IRB approval and written consent was obtained prior to participation.

\section{Instruments}

ES was delivered using the Respond Select® (Empi, Inc., St. Paul, Minnesota) neuromuscular electrical stimulation system. Self-adhesive, reusable, latex-free bipolar stimulating surface electrodes $(90 \times 50 \mathrm{~mm}$ square for $\mathrm{VL} ; 3.5 \mathrm{~cm}$ round for $\mathrm{APB}$ ) were placed on the skin following Empi, Inc. guidelines (Figure 1 - rightside images) and connected to the ES device for muscle activation. EMG signals were acquired through the Nexus-10 EMG device (MindMedia B.V., Netherlands). Sampling frequency was set at $2048 \mathrm{~Hz}$. Pre-gelled, selfadhesive $\mathrm{Ag} / \mathrm{AgCl}$ EMG surface electrodes were placed across the muscle belly in parallel with the muscle fibers according to Surface ElectroMyoGraphy for the Non-Invasive Assessment of Muscles (SENIAM) guidelines. Isometric forces for the APB were measured using the PasPort high resolution force sensor (range: \pm $50 \mathrm{~N}$, resolution: $0.002 \mathrm{~N}$; PASCO Scientific Inc., Roseville, CA) and for the VL using the Shimpo Instruments (ELECTROMATIC Equipment Co., Cedarhurst, NY) Javelin FGV-HXY Force Gauge (maximal capacity: 500lb/250kg; accuracy: +/-0.2\% F.S.).

\section{Experimental Set-up}

Protocols were developed for the APB and VL to ensure stabilization and isolation of the related muscles during testing. For APB testing, subjects were seated with the arm in $90^{\circ}$ elbow flexion. Hand and forearm muscles were isolated via resting on a board that blocks the fingers, only to let the thumb contract. ${ }^{21}$ The thumb force sensor was affixed to a rod projecting from the table surface so as to be positioned facing the palm of the hand at thumb height (Figure 1a). For VL testing, subjects were seated in an upright chair (hips at 90 flexion) as such that feet did not make contact with the ground and the back of the knee joint contacted the edge of the chair. A strap was placed firmly around the waist to isolate the quadriceps. ${ }^{21,22}$ The force sensor was positioned facing the lower anterior leg, and was affixed from the side of the chair (Figure 1b).

\section{Procedure}

The order at which the frequencies were delivered and of which muscle was first stimulated was randomized. During an acclimation session, each participant was familiarized with appropriate posturing and execution of MVC. Furthermore, each participant was familiarized with ES sensation to ensure they felt comfortable during the actual procedure.

\section{Part One: Pre-fatigue MVC}

For each muscle at each of three stimulation frequencies $(10,35$, and $50 \mathrm{~Hz})$, all participants performed two prefatigue MVC trials (holding contraction for 5 seconds then relaxing) of isometric knee and thumb extension. Muscle force and EMG was simultaneously recorded. Verbal encouragement was offered from the rater. A minimum of 30 seconds of rest was given between the

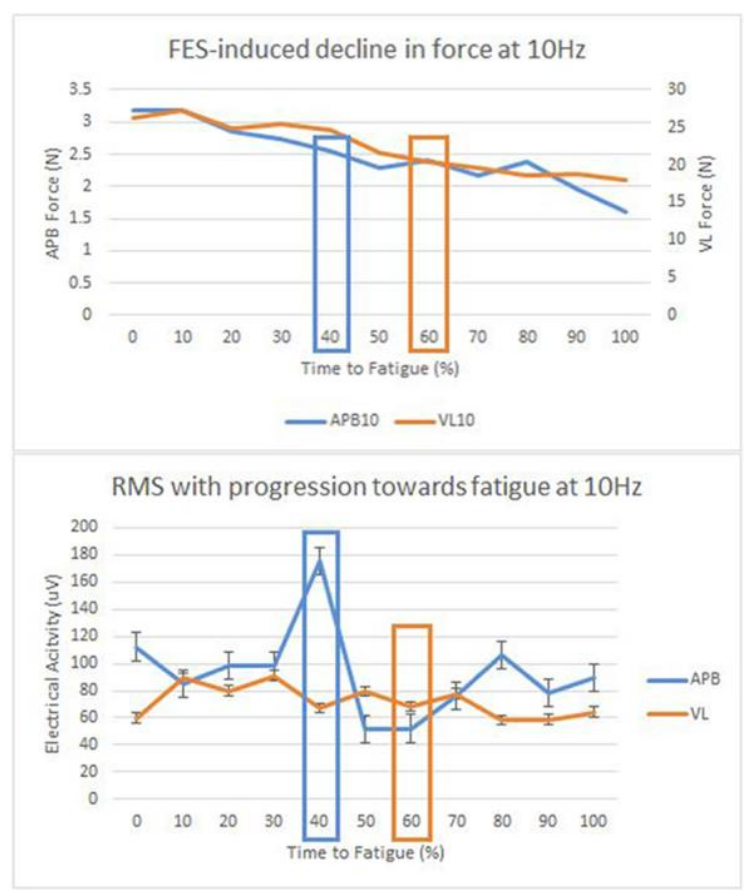

Fig 3.Force and electrical activity changes during ES at $10 \mathrm{~Hz}$ with progression towards fatigue. 


\section{ES Frequency: Effects on Muscle Force and Fatigue}

Eur J Transl Myol 27 (4): 239-245

two trials to allow adequate recovery. The MVC values were averaged and recorded as the preMVC value. The force measure that equated to $25 \%$ preMVC was then calculated and a contraction to $25 \% \mathrm{MVC}$ was performed while the EMG was recorded. The EMG signals were then used for comparison with EMG signals obtained during ES-induced contraction to $25 \% \mathrm{MVC}$ following EMD filtering.

\section{Part Two: ES-induced progression towards fatigue}

Prior to the fatiguing protocol for a given ES frequency, each participant's required stimulation current intensity to achieve $25 \%$ preMVC force through ES-induced contraction was determined. This value was then set as a constant for the fatiguing protocol and the timer began to record time to fatigue. Each participant was stimulated at a randomized frequency order $(10,35$, and $50 \mathrm{~Hz}$ ) with rectangular, biphasic pulses delivered at pulse-widths of $300 \mathrm{us}$. Pulses were delivered at a sequence of $4 \mathrm{sON} / 4 \mathrm{sOFF}{ }^{14}$ Stimulation concluded when the muscle fatigued, defined as a $50 \%$ drop in force from the initial $25 \%$ preMVC force for three consecutive contractions, ${ }^{14}$ or at the expiration of 30 minutes of ES. EMG was recorded throughout the protocol. The timer stopped once fatigue or 30 minutes was reached. At least 30 minutes of rest was given inbetween fatiguing protocols for each muscle.

\section{Signal Analysis}

All EMG signals were initially filtered using a Butterworth filter $\left(20-500 \mathrm{~Hz}\right.$ cutoff, $5^{\text {th }}$-order $)$ inherent in the device's BioTrace+ software (MindMedia B.V.,

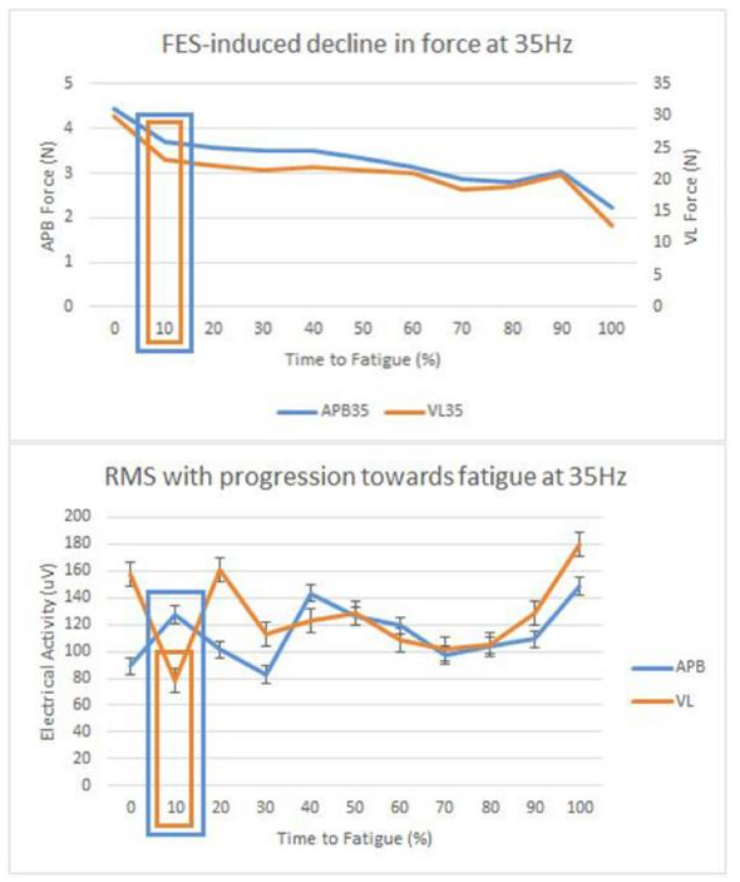

Fig. 4. Force and electrical activity changes during $\mathrm{ES}$ at $35 \mathrm{~Hz}$ with progression towards fatigue.
Netherlands). Data was then transferred to MATLAB (The MathWorks, Inc., Natick, Massachusetts) for further processing. The middle two seconds of ESinduced EMG data was extracted at 11 time points, denoted as $\%$ of total time to fatigue (TTF), for each participant (initial, 10, 20, 30, 40, 50, 60, 70, 80, 90, and $100 \%$ of TTF). EMD was applied to all ES-induced signal data. EMD was previously described in Pilkar et al. ${ }^{19}$ RMS averaging was performed on all ES signals (post-EMD filtered). EMD-filtered ES signals were also full-wave rectified and noise minimized with a $4^{\text {th }}$-order, zero-lag, low-pass filter at $5 \mathrm{~Hz}$ cutoff frequency to form a linear envelope. From the linear envelope, integrated EMG (iEMG) was calculated. Additionally, the Fast Fourier Transform was applied to the full-wave rectified signal and the MDF was calculated.

\section{Statistical Analysis}

After testing for normality, ES current intensities (mA) required to reach $25 \% \mathrm{MVC}$ and changes in ES-induced force and EMG parameters at each time point were evaluated for statistical significance using ANOVAs and Tukey post-hoc tests. Values are reported as mean \pm standard error. Significance was defined by a p-value set at $p<0.05$. SAS V9.4 was used for all statistical analysis.

\section{Results}

\section{ES Current Intensity}

Generally, the APB required lower initial ES current intensity to achieve the required force (25\%MVC) compared to the VL (mean APB: $15.77 \pm 1.15 \mathrm{~mA}$, mean VL: $46.77 \pm 1.15 \mathrm{~mA}, \mathrm{p}<0.0001)$ for all frequencies. The required current intensity to achieve force at $10 \mathrm{~Hz}$ was significantly greater $(\mathrm{p}<0.0001)$ than at $35 \mathrm{~Hz}$ or $50 \mathrm{~Hz}$ for the APB (mean at $10 \mathrm{~Hz}: 20.9 \pm 0.97 \mathrm{~mA}$, mean at $35 \mathrm{~Hz}: 13.3 \pm 1.14 \mathrm{~mA}$, mean at $50 \mathrm{~Hz}: 13.1 \pm 0.96 \mathrm{~mA})$ and non-significantly greater for the $\mathrm{VL}$ (mean at $10 \mathrm{~Hz}$ : $51.4 \pm 2.42 \mathrm{~mA}$, mean at $35 \mathrm{~Hz}: 44.9 \pm 2.85 \mathrm{~mA}$, mean at $50 \mathrm{~Hz}: 44.0 \pm 2.60 \mathrm{~mA})$. The muscle type*frequency interaction effect significantly influenced stimulation intensity $(\mathrm{p}<0.0001)$ in all cases. For example, APB at $10 \mathrm{~Hz}$ differed significantly from VL at 10,35 , and $50 \mathrm{~Hz}$ as did APB at $35 \mathrm{~Hz}$ and APB at $50 \mathrm{~Hz}$ (Figure 2).

\section{ES-induced Fatigue}

Overall, when combining all frequencies for both muscles, force significantly declined with progression of ES-induced contraction $(\mathrm{p}<0.0001)$, indicating muscle fatigue. Comparisons between frequencies and progression of fatigue for each muscle indicated that ES of the APB at 10,35 , and $50 \mathrm{~Hz}$ lead to significant force decline after $40 \%, 10 \%$, and $70 \% \mathrm{TTF}$, respectively for each frequency. Corresponding to these points (40\%, $10 \%$, and $70 \% \mathrm{TTF}$ ) of significant force decline, a sudden increase in EMG amplitude was observed. Observations in the VL at 10,35 , and $50 \mathrm{~Hz}$ revealed significant force decline after $60 \%, 10 \%$, and $80 \% \mathrm{TTF}$, 


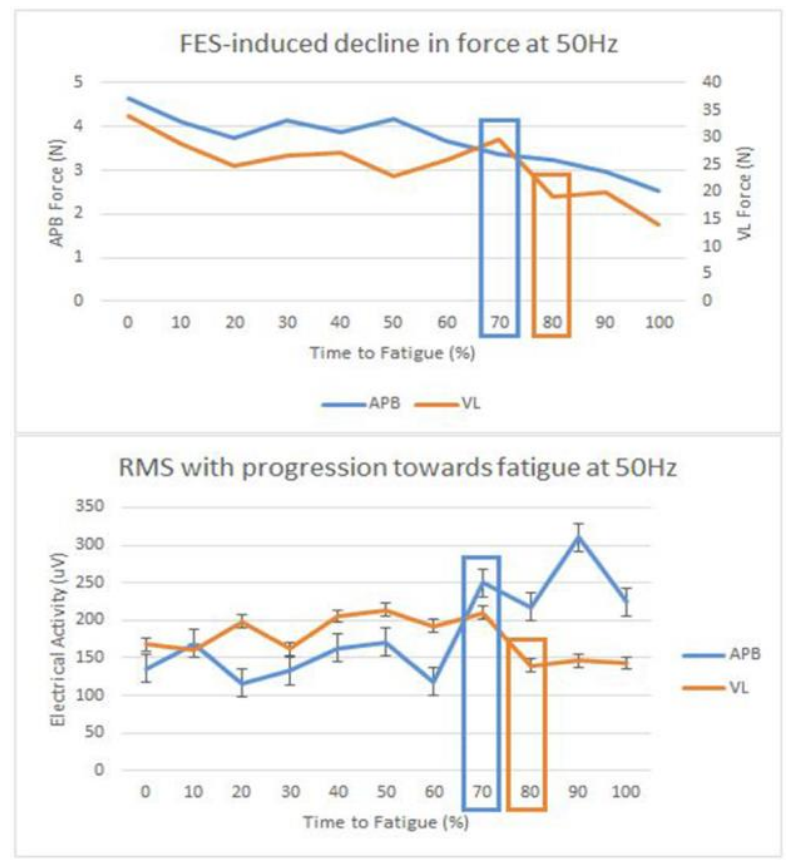

Fig 5. Force and electrical activity changes during ES at $50 \mathrm{~Hz}$ with progression towards fatigue.

respectively for each frequency. Corresponding to these points $(60 \%, 10 \%$, and $80 \% \mathrm{TTF})$ of significant force decline, a sudden decrease in EMG amplitude was observed. Figures 3-5 display the respective electrical activity shifts for each muscle during stimulation at each frequency.

\section{Discussion}

Electrical stimulation is applied during rehabilitation to promote functional gains through increased force generation and improved muscle integrity. In addition to the reported effects of increased cell proliferation and angiogenesis, ${ }^{19,23}$ enhanced muscle integrity and force generation is further achieved by the ES-induced exercise-like movement that leads to fiber type transformation and increased muscle cross-sectional area. $^{20,24-31}$ With this knowledge, the aim of this investigation was to better understand how different muscle fiber compositions can impact optimal ES performance, particularly to delay fatigue development. Research comparing the application of ES on muscles of varying fiber proportion in the same group of subjects is limited. The result of our study supports our hypothesis that the frequency of ES may play a role in force generation and fatigue development of muscles irrespective of size and characteristics. Further, muscles of different size and fiber composition, seem to behave differently as fatigue progresses. The results show that for both the APB and VL there was a trend in requiring higher ES current intensity during lower frequency $(10 \mathrm{~Hz})$ in order to achieve the initial force (in our study $25 \% \mathrm{MVC}$ ). Furthermore, the initial ES intensity required to achieve $25 \% \mathrm{MVC}$ was significantly greater for the VL muscle compared to APB. This difference can be explained by the greater proportion of type II fibers, which have higher firing thresholds and larger diameters, in the VL. Kuriki et al. reported that muscle control differs between sizes. ${ }^{32}$ Small muscles, like the $\mathrm{APB}$, assist in fine motor movements by innervating fewer fibers per motor unit; therefore, they are less force producing in exchange for greater precision. In contrast, large muscles, like the VL, assist in gross motor function, generating significantly greater forces than smaller muscles by innervating upwards of 100 to 1000 fibers per motor unit. ${ }^{33}$ Therefore, greater intensity is necessary to activate the larger motor units of the large muscle. Moreover, in the VL, it was reported that recruitment thresholds range from $\sim 5.5$ to 22.7 pulses per second ${ }^{34}$ and given that more fast-twitch fibers tend to be superficially located, ${ }^{12}$ minimal motor unit recruitment will be achieved at low-levels of stimulation frequency compared to 35 and $50 \mathrm{~Hz}$, the latter two which surpass the recruitment threshold for many motor units. Since the APB contains lower-threshold motor units, the recruitment threshold range suggests that stimulation delivered at frequencies above $10 \mathrm{~Hz}$ will greatly maximize recruitment and therefore contribute to intensity being less influenced by frequency. In conclusion, the results of our investigation indicated that an association exists between ES-induced force decline and the relative EMG responses. The corresponding sudden increase in electrical activity of the APB and decrease in electrical activity of the VL when ESinduced force declined may be due to the better glycogen storage maintenance and utilization ability of type I fibers. Gregory et al. evaluated the influence of metabolic characteristics and phenotypic expression of individual fibers in predicting glycogen utilization during ES. ${ }^{34}$ Outcomes demonstrated that both an enzyme ratio of succinate dehydrogenase and quantitative-actomyosin ATPase (SDH:qATPase) and fiber phenotype significantly predicted glycogen utilization, where type I fibers are the most proficient at utilizing and maintaining glycogen stores. ${ }^{34}$ Therefore, it is possible that the fibers of the APB are capable of producing a sudden electrical activity increase at the moment of significant force decline as a final mechanism attempting to sustain force before fatigue while the fast-fatiguing type II fibers do not sustain the same energy capacity and thus, the electrical activity drops at the moment of significant force decline. Furthermore, studies have shown that to increase muscle strength the force production should be maintained at or above $60 \%$ MVC. Though our research only stimulated to $25 \% \mathrm{MVC}$, it appears that higher frequencies such as $50 \mathrm{~Hz}$ could be used to increase muscle strength. This is suggested because ES at $10 \mathrm{~Hz}$, particularly for the small $\mathrm{APB}$, required increases in intensity beyond some participants' pain thresholds and therefore may not be tolerable, but the higher frequencies were more comfortable and able to recruit more motor units. Thus, 
lower frequencies may be more applicable to improve muscle integrity without intention to increase force and higher frequencies may be suitable to promote increases in force and muscle strength. The observed relationship between the initial moment of significant force decline and shift in electrical activity should assist clinicians and researchers when developing ES protocols to minimize fatigue based on muscle size and fiber composition. Based on significant differences observed between muscle force decline and ES frequencies at 10, 35 and $50 \mathrm{~Hz}$, we propose to utilize higher frequencies for increasing muscle force (for example in athletic training) and lower frequencies due to their slower onset of fatigue for rehabilitation programs in which force is not the main outcome. The findings from this investigation may provide some guidelines for health professionals to adjust the ES current intensity based on fatigue development to encourage the recruitment of additional motor units to sustain force generation and prevent early fatigue.

\section{List of acronyms}

APB - abductor pollicis brevis

EMD - empirical mode decomposition

EMG - electromyography

ES - electrical stimulation

IMFs - intrinsic mode functions

IRB - Institutional Review Board

$\mathrm{MNF}$ - mean frequency

MVC - maximal voluntary contraction

qATPase - quantitative-actomyosin ATPase

RMS - root-mean-square

SD - succinate dehydrogenase

SENIAM - Non-Invasive Assessment of Muscles

TTF - time to fatigue

VL - vastus lateralis

\section{Author's contributions}

MV and PF equally participated in experimental design, data collection, writing and revision of the manuscript.

\section{Acknowledgments}

Funding Disclosure: This work is supported in part by the National Science Foundation under EFRI Grant 1332329. We would like to thank Magdalena Wegrzyniak and Sarina Moghadam for their help with data collection and the School of Engineering machine shop for their aid in constructing dynamometer-holding devices.

\section{Conflict of Interest}

The authors have no conflicts of interests.

\section{Ethical Publication Statement}

We confirm that we have read the Journal's position on issues involved in ethical publication and affirm that this report is consistent with those guidelines.

\section{Corresponding Author}

Pouran Faghri, UConn, Department of Allied Health Sciences, 358 Mansfield Road, U-2101, Koons Hall Room 318, Storrs, CT, USA 06269-1101; Phone: 860486-0018, Fax:-5375. E-mail: pouran.faghri@uconn.edu

\section{E-mails of co-author}

Maria Vromans: maria.vromans@uconn.edu

\section{References}

1. Gregory C, Dixon W, Bickel C. Impact of varying pulse frequency and duration on muscle torque production and fatigue. Muscle Nerve 2007;35:504-9.

2. Chesler N, Durfee W. Surface EMG as a fatigue indicator during FES-induced isometric muscle contractions. J Electromyogr Kinesiol 1997;7:2737.

3. Papaiordanidou M, Guiraud D, Varray A. Does central fatigue exist under low-frequency stimulation of a low fatigue-resistant muscle?. Eur J Appl Phsiol 2010;110:815-23.

4. Alexandre F, Derosiere G, Papaiordanidou M, et al. Cortical motor output decreases after neuromuscular fatigue induced by electrical stimulation of the plantar flexor muscles. Acta Physiol 2015;214:124-34.

5. Estigoni E, Fornusek C, Hamzaid N, et al. Evoked EMG versus muscle torque during fatiguing functional electrical stimulation-evoked muscle contractions and short-term recovery in individuals with spinal cord injury. Sensors 2014;14:2290720.

6. Hamada T, Kimura T, Moritani T. Selective fatigue of fast motor units after electrically elicited muscle contractions. J Electromyogr Kinesiol 2004;14:531-8.

7. Johnson K, Edwards S, Van Tongeren C, Bawa P. Properties of human motor units after prolonged activity at a constant firing rate. Exp Brain Res 2003;154:479-87.

8. Ibitoye M, Estigoni E, Hamzaid $\mathrm{N}$, et al. The effectiveness of FES-evoked EMG potentials to assess muscle force and fatigue in individuals with spinal cord injury. Sensors 2014;14:12598-622.

9. Andrade A, Nasuto S, Kyberd P, et al. EMG signal filtering based on empirical mode decomposition. Biomed Signal Process Control 2006;1:44-55.

10. Zhang D, Ang WT. Reciprocal EMG controlled FES for pathological tremor suppression of forearm. Conf Proc IEEE Eng Med Biol Soc 2007.

11. Pilkar RB, Yarossi M, Forrest G. Empirical mode decomposition as a tool to remove the function electrical stimulation artifact from surface electromyograms: preliminary investigation. Conf Proc IEEE Eng Med Biol Soc 2012;1847-50. 
12. Polgar J, Johnson M, Weightman D, Appleton D. Data on fibre size in thirty-six human muscles. J Neurol Sci 1973;19:307-18.

13. Andrzejewska R, Jaskólski A, Jaskólska A, et al. Electromyogram features during linear torque decrement and their changes with fatigue. Eur $\mathbf{J}$ Appl Phsiol 2014;114:2105-17.

14. Behringer M, Grützner S, Montag J, et al. Effects of stimulation frequency, amplitude, and impulse width on muscle fatigue. Muscle Nerve 2015;53:608-16.

15. Dreibati B, Lavet C, Pinti A, Poumarat G. Influence of electrical stimulation frequency on skeletal muscle force and fatigue. Ann Phys Rehabil Med 2010;53:266-77.

16. Wegrzyk J, Fouré A, Le Fur Y, et al. Responders to wide-pulse, high-frequency neuromuscular electrical stimulation show reduced metabolic demand: A 31P-MRS study in humans. PloS One 2015;10:e0143972.

17. Bickel C, Slade J, VanHiel L, et al. Variablefrequency-train stimulation of skeletal muscle after spinal cord injury. J Rehabil Res Dev Clin Suppl 2004;41:33.

18. Chou L, Binder-Macleod S. The effects of stimulation frequency and fatigue on the forceintensity relationship for human skeletal muscle. Clin Neurophysiol 2007;118:1387-96.

19. Maffulli N, Furia JP. Rotator cuff disorders: Basic science and clinical medicine. JP Medical Ltd 2012.

20. Kern H, Boncompagni S, Rossini K, et al. Longterm denervation in humans causes degeneration of both contractile and excitation-contraction coupling apparatus, which is reversible by functional electrical stimulation (FES): A role for myofiber regeneration? J Neuropathol Exp Neurol 2004;63:919-31.

21. Stratton K, Faghri P. Electrically and hybridinduced muscle activations: effects of muscle size and fiber type. Eur J Transl Myol 2016;26:6163. eCollection 2016 Jun 13.

22. Bohannon R, Kindig J, Sabo G, et al. Isomeric knee extension force measured using a handheld dynamometer with and without belt-stabilization. Physiother Theory Pract 2011;28:562-8.

23. Kanno S, Oda N, Abe M, et al. Establishment of a simple and practical procedure applicable to therapeutic angiogenesis. Circulation 1999;99:2682-7.

24. Andersen JL, Mohr T, Biering-Sørensen F, et al. Myosin heavy chain isoform transformation in single fibres from $\mathrm{m}$. vastus lateralis in spinal cord injured individuals: Effects of long-term functional electrical stimulation (FES). Pflügers Arch 1996;431:513-8.

25. Crameri R, Weston A, Climstein M, et al. Effects of electrical stimulation-induced leg training on skeletal muscle adaptability in spinal cord injury. Scand J Med Sci Sports 2002;12:316-22.

26. Boncompagni S, Kern H, Rossini $\mathrm{K}$, et al. Structural differentiation of skeletal muscle fibers in the absence of innervation in humans. Proc Natl Acad Sci USA. 2007;104:19339-44.

27. Kern H, Carraro U, Adami N, et al. One year of home-based functional electrical stimulation (FES) in complete lower motor neuron paraplegia: recovery of tetanic contractility drives the structural improvements of denervated muscle. Neurol Res. 2010;32:5-12. DOI:1 $0.1189 / 184313209 X 385644$

28. Kern H, Carraro U, Adami N, et al. Home-based functional electrical stimulation rescues permanently denervated muscles in paraplegic patients with complete lower motor neuron lesion. Neurorehabil Neural Repair 2010;24:709-21. Doi 10.1177/1545968310366129. Epub 2010 May 11.

29. Kern H, Carraro U. Home-based functional electrical stimulation (h-b FES) for long- term denervated human muscle: history, basics, results and perspectives of the Vienna rehabilitation strategy. Eur J Transl Myol. 2014;24:27-40.

30. Carraro U, Kern H, Gava P, et al. Biology of Muscle Atrophy and of its Recovery by FES in Aging and Mobility Impairments: Roots and ByProducts. Eur J Transl Myol 2015;25:221-30. doi: 10.4081/ejtm.2015.5272.

31. Kern H, Hofer C, Loefler S, et al. Atrophy, ultrastructural disorders, severe atrophy and degeneration of denervated human muscle in SCI and Aging. Implications for their recovery by Functional Electrical Stimulation, updated 2017. Neurol Res 2017;39:660-6. doi: 10.1080/01616412 .2017.1314906. Epub 2017 Apr 13.

32. Kuriki HU, De Azevedo FM, Takahashi LSO, et al. The relationship between electromyography and muscle force: EMG methods for evaluating muscle and nerve function. InTech 2012.

33. Trevino M, Herda T, Fry A, et al. Influence of the contractile properties of muscle on motor unit firing rates during a moderate-intensity contraction in vivo. J Neurophysiol 2016;116:552-62.

34. Gregory CM, Williams RH, Vandenborne K, Dudley GA. Metabolic and phenotypic characteristics of human skeletal muscle fibers as predictors of glycogen utilization during electrical stimulation. Euro J Appl Physiol 2005;95:276-82. 